\title{
GALOIS-STABILITY FOR TAME ABSTRACT ELEMENTARY CLASSES
}

\author{
RAMI GROSSBERG AND MONICA VANDIEREN
}

\begin{abstract}
We introduce tame abstract elementary classes as a generalization of all cases of abstract elementary classes that are known to permit development of stability-like theory. In this paper we explore stability results in this new context. We assume that $\mathcal{K}$ is a tame abstract elementary class satisfying the amalgamation property with no
\end{abstract} maximal model. The main results include:

Theorem 0.1. Suppose that $\mathcal{K}$ is not only tame, but $\mathrm{LS}(\mathcal{K})$-tame. If $\mu \geq \operatorname{Hanf}(\mathcal{K})$ and $\mathcal{K}$ is Galois stable in $\mu$, then $\kappa_{\mu}(\mathcal{K})<\beth_{\left(2^{\operatorname{Hanf}(\mathcal{K})}\right)^{+}}$, where $\kappa_{\mu}(\mathcal{K})$ is a relative of $\kappa(T)$ from first order logic.

$\operatorname{Hanf}(\mathcal{K})$ is the Hanf number of the class $\mathcal{K}$. It is known that $\operatorname{Hanf}(\mathcal{K}) \leq$ $\left.\beth_{\left({ }^{\mathrm{LS}}(\mathcal{K})\right.}\right)+$

The theorem generalizes a result from Sh3. It is used to prove both the existence of Morley sequences for non-splitting (improving Claim 4.15 of [Sh 394] and a result from GrLe1]) and the following initial step towards a stability spectrum theorem for tame classes:

Theorem 0.2. If $\mathcal{K}$ is Galois-stable in some $\mu>\beth_{\left({ }_{2} \mathrm{Hanf}(\mathcal{K})\right)^{+}}$, then $\mathcal{K}$ is stable in every $\kappa$ with $\kappa^{\mu}=\kappa$. E.g. under $\mathrm{GCH}$ we have that $\mathcal{K}$ Galois-stable in $\mu$ implies that $\mathcal{K}$ is Galois-stable in $\mu^{+n}$ for all $n<\omega$.

\section{INTRODUCTION}

In the last twenty years most of Shelah's effort in model theory was in developing classification theory for abstract elementary classes (Some important mile stones are: Sh 87a], Sh 87b, Sh 88], Sh 300, Sh 394], Sh 576, Sh 600 and Sh 705]). An abstract elementary class (AEC) is a class of structures of the same similarity type endowed with a morphism satisfying natural properties such as closure under directed limits (see Gr1 for an introduction). The notion of an AEC (introduced by Shelah in Sh 88) is

Date: September 22, 2005.

AMS Subject Classification: Primary: 03C45, 03C52, 03C75. Secondary: 03C05, $03 \mathrm{C} 55$ and $03 \mathrm{C} 95$.

This paper is part of the second author's Ph.D. thesis written under the guidance of Rami Grossberg. 
broad enough to capture classes axiomatized by non-first-order logics including $L_{\omega_{1}, \omega}(\mathbf{Q})$. To date, there is no known stability theory or even a categoricity theorem for abstract elementary classes without some additional strong assumptions.

The most general of Shelah's attempts to develop a stability theory is Sh 600] where Shelah works in the context of AECs with the amalgamation and joint embedding properties and a very nicely behaved forking-like relation. He introduces the notion of good-frame which is a generalization of a first-order superstable theory (the definition alone needs a couple of pages). Most of his 100-page paper is dedicated to the derivation of structural results under the assumption that the AEC has a forking-like relation.

In our paper we deal with a much wider class of AECs than Shelah in Sh 600 as we do not assume as part of the setting built-in forking and regular types. We deal with stable but tame classes (see Definitions 1.7 and (3.2), in other words, stable classes where inequality of types behaves locally. Tameness is a general property capturing finite diagrams (homogeneous model theory). While it is not difficult to see that excellent classes of atomic models of a first-order theory (from Sh 87a and Sh 87b]) are tame, Grossberg and Alexei Kolesnikov show that every abstract excellent class is also tame (see GrKo]). Very recently using ideas from GrKo, Andrés Villaveces and Pedro Zambrano discovered that the class of structures obtained from Hrushovski's fusion of strongly minimal first-order theories is another source of examples of tame AECs. See [ViZa] and $[\mathrm{Ba}]$.

Despite the generality of our context, recent progress on a Morley's Theorem for tame abstract elementary classes provides evidence that a stabilitytheory can be developed. A short time after submitting this paper for publication we realized that using the splitting machinery developed here, we can prove in ZFC an instance of Shelah's categoricity conjecture for tame classes (see GrVa1 and GrVa2]).

Theorem 0.3 (Grossberg and VanDieren 2003). Let $\mathcal{K}$ be an $A E C, \kappa:=$ $\beth_{\left(2^{\mathrm{LS}(\mathcal{K})}\right)^{+}}$. Denote by $\mu_{0}:=\beth_{\left(2^{\kappa}\right)^{+}}$. Suppose that $\mathcal{K}_{>\kappa}$ has the amalgamation property and is $\kappa$-tame. If $\mathcal{K}$ is categorical in some $\lambda^{+}>\mu_{0}$ then $\mathcal{K}$ is categorical in every $\mu \geq \mu_{0}$.

This work removes the set-theoretic assumption of a strongly compact cardinal from the work of Makkai and Shelah [MaSh.

In addition to examining splitting in this paper, we prove the existence of Morley sequences from the assumption of stability in tame AECs. We plan to use Morley sequences for non-splitting to define a dividing-like concept for these classes and to prove a stability spectrum theorem for AECs. More work on the stability spectrum theorem (extension of our results under the additional assumption of $\operatorname{LS}(\mathcal{K})=\aleph_{0}$ ) appears in BaKuVa.

We are grateful to John Baldwin for reading several preliminary versions and suggesting us to consider the stability spectrum problem for AECs. 
The referee's comments and questions led to significant improvement of the presentation.

\section{BACKGROUND}

Much of the necessary background for this paper can be found in Gr1]. We will use $\alpha, \beta, \gamma, i, j$ to denote ordinals and $\kappa, \lambda, \mu, \chi$ will be used for cardinals. We will use $(\mathcal{K}, \prec \mathcal{K})$ to denote an abstract elementary class and $\mathcal{K}_{\mu}$ is the subclass of models in $\mathcal{K}$ of cardinality $\mu$. Similarly we define $\mathcal{K}_{<\mu}$ and $\mathcal{K}_{\geq \mu}$. Models are denoted by $M, N$ and may be decorated with superscripts and subscripts. Sequences of elements from $M$ are written as $\bar{a}, \bar{b}, \bar{c}, \bar{d}$ and we write ${ }^{\beta} \mathrm{M}$ to denote the collection of all sequences of length $\beta$ made up of elements from $M$. The letters $e, f, g, h$ are reserved for $\mathcal{K}$ mappings and id is the identity mapping.

For the remainder of this paper we will fix $(\mathcal{K}, \prec \mathcal{K})$ to be an abstract elementary class.

We begin by reviewing the definition of Galois-type, since we will be considering variations of the underlying equivalence relation $E$ later in this paper.

Definition 1.1. Let $\beta>0$ be an ordinal. For triples $\left(\bar{a}_{\ell}, M, N_{l}\right)$ where $\bar{a}_{\ell} \in{ }^{\beta} \mathrm{N}_{\ell}$ and $M_{\ell} \prec \mathcal{K} N_{\ell} \in \mathcal{K}$ for $\ell=0$, 1, we define a binary relation $E$ as follows: $\left(\bar{a}_{0}, M, N_{0}\right) E\left(\bar{a}_{1}, M, N_{1}\right)$ iff and there exists $N \in \mathcal{K}$ and $\mathcal{K}$-mappings $f_{0}, f_{1}$ such that $f_{\ell}: N_{\ell} \rightarrow N, f_{\ell} \uparrow M=\operatorname{id}_{M}$ for $\ell=0,1$ and $f_{0}\left(\bar{a}_{0}\right)=f_{1}\left(\bar{a}_{1}\right)$ :

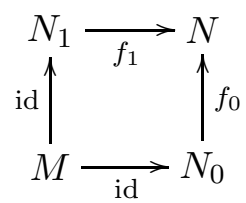

The relation $E$ is used to define Galois-types in classes that satisfy the amalgamation property.

Definition 1.2. (1) Let $\mu \geq \operatorname{LS}(\mathcal{K})$. We say that $\mathcal{K}$ has the $\mu$-amalgamation property iff for any $M_{\ell} \in \mathcal{K}_{\mu}($ for $\ell \in\{0,1,2\})$ such that $M_{0} \prec_{\mathcal{K}} M_{1}$ and $M_{0} \prec_{\mathcal{K}} M_{2}$ there are $N \in \mathcal{K}_{\mu}$ and $\mathcal{K}$ embeddings $f_{\ell}: M_{\ell} \rightarrow N$ such that $f_{\ell} \uparrow M_{0}=\operatorname{id}_{M_{0}}$ for $\ell=1,2$.

(2) A model $M_{0} \in \mathcal{K}_{\mu}$ satisfying the above requirement is called an amalgamation base.

(3) $\mathcal{K}$ has the amalgamation property iff $\mathcal{K}$ has the $\mu$-amalgamation property for all $\mu \geq \operatorname{LS}(\mathcal{K})$.

Remark 1.3. $E$ is an equivalence relation on the class of triples of the form $(\bar{a}, M, N)$ where $M \prec_{\mathcal{K}} N, \bar{a} \in N$ and both $M$ and $N$ are amalgamation bases. When $N$ is not an amalgamation base, $E$ may fail to be transitive, but the transitive closure of $E$ could be used instead. 
Remark 1.4. While, the focus of this paper is on classes with the amalgamation property, several of the proofs in this paper can be adjusted to the context of abstract elementary classes with density of amalgamation bases as in ShVi] and Va].

We will make the following assumption for the remainder of the paper:

Assumption 1.5. $\mathcal{K}$ satisfies the amalgamation property.

Under the assumption of the amalgamation property, there exists a large model-homogeneous model, which we will denote by $\mathfrak{C}$ and call the monster model. A $\mu$-model homogeneous model is a model $M$ in which for every $N \prec_{\mathcal{K}} M$ of cardinality $\mu$ and every extension $N_{1}$ of cardinality $\mu$, there exists a $\mathcal{K}$-embedding $f: N_{1} \rightarrow M$ with $f \uparrow N=\mathrm{id}_{N}$. $M$ is model homogeneous when it is $\mu$-model homogeneous for every $\mu<\|\mathfrak{C}\|$. All models and sequences of elements will be assumed to come from $\mathfrak{C}$. Thus, $E$ is an equivalence relation and we can now define types as equivalence classes of E.

In order to avoid confusing this non-elementary notion of "type" with the conventional first-order one (i.e. set of formulas) we will follow Gr1] and Gr2] and introduce it below under the name of Galois-type.

Definition 1.6. Let $\beta$ be a positive ordinal (can be one).

(1) For $M, N \in \mathcal{K}$ and $\bar{a} \in{ }^{\beta} \mathrm{N}$. The Galois-type of $\bar{a}$ in $N$ over $M$, written ga-tp $(\bar{a} / M, N)$, is defined to be $(\bar{a}, M, N) / E$.

(2) We abbreviate ga-tp $(\bar{a} / M, N)$ by ga-tp $(\bar{a} / M)$.

(3) For $M \in \mathcal{K}$,

$$
\operatorname{ga-S}^{\beta}(M):=\left\{\operatorname{ga-tp}(\bar{a} / M, N) \mid M \prec N \in \mathcal{K}_{\|M\|}, \bar{a} \in{ }^{\beta} \mathrm{N}\right\} .
$$

We write ga- $\mathrm{S}(M)$ for ga- $\mathrm{S}^{1}(M)$.

(4) Let $p:=\operatorname{ga-} \operatorname{tp}\left(\bar{a} / M^{\prime}, N\right)$ for $M \prec \mathcal{K} M^{\prime}$ we denote by $p\lceil M$ the type $\operatorname{ga-tp}(\bar{a} / M, N)$. The domain of $p$ is denoted by $\operatorname{dom}(p)$ and it is by definition $M^{\prime}$.

(5) Let $p=\operatorname{ga-tp}(\bar{a} / M, N)$, suppose that $M \prec \mathcal{K} N^{\prime} \prec \mathcal{K} N$ and let $\bar{b} \in{ }^{\beta} \mathrm{N}^{\prime}$ we say that $\bar{b}$ realizes $p$ iff ga-tp $\left(\bar{b} / M, N^{\prime}\right)=p\lceil M$.

(6) For types $p$ and $q$, we write $p \leq q$ if $\operatorname{dom}(p) \subseteq \operatorname{dom}(q)$ and there exists $\bar{a}$ realizing $p$ in some $N$ extending $\operatorname{dom}(p)$ such that $(\bar{a}, \operatorname{dom}(p), N)=$ $q \uparrow \operatorname{dom}(p)$.

Definition 1.7. We say that $\mathcal{K}$ is $\beta$-Galois-stable in $\mu$ if for every $M \in \mathcal{K}_{\mu}$, $\mid$ ga- $\mathrm{S}^{\beta}(M) \mid=\mu$. The class $\mathcal{K}$ is Galois stable in $\mu$ iff $\mathcal{K}$ is 1 -stable in $\mu$.

Remark 1.8. Let $T$ be a stable, countable, first-order, complete theory. Set $\mathcal{K}:=\operatorname{Mod}(T)$ and $\prec_{\mathcal{K}}$ the usual elementary submodel relation. Take $\mu=2^{\aleph_{0}}$. While $\mathcal{K}$ is 1 -Galois-stable in $\mu$ (in fact it is $n$-Galois-stable in $\mu$ for all $n<\omega)$, it can be shown that $\mathcal{K}$ is $\omega$-Galois-stable in $\mu$ iff $\mathcal{K}$ has the ndop and notop. 
While there is a nice relationship between $\beta$-stability and $\beta^{\prime}$-stability for first order theories, the corresponding relationship between $\beta$-stability and $\beta^{\prime}$-stability for AECs in general is unknown and probably fails.

Notation 1.9. Although not standard in AECs with the amalgamation property, we will use the notation ga-tp $(\bar{a} / \emptyset)$ to denote the orbit of $\bar{a}$ under Aut $(\mathfrak{C})$. This notation is only used as a device in the proof of Theorem 4.13

\section{Saturated ANd Limit Models}

In this section we recall that limit models (see Definition 2.6) can be used as a substitute for saturated models. Later, we will see that limit models will be more convenient in situations where the first order case would call for saturated models.

Definition 2.1. We say that $M \in \mathcal{K}_{>\mathrm{LS}(\mathcal{K})}$ is Galois-saturated if for every $N \prec \mathcal{K} M$ of cardinality $<\|M\|$, and every $p \in$ ga-S $(N)$, we have that $M$ realizes $p$.

Remark 2.2. When $\mathcal{K}=\operatorname{Mod}(T)$ for a first-order $T$, using the compactness theorem one can show (Theorem 2.2.3 of [Gr1]) that for $M \in \mathcal{K}$, the model $M$ is Galois-saturated iff $M$ is saturated in the first-order sense.

We begin with universal extensions which are used to build limit models. A universal extension captures some properties of saturated models without referring explicitly to types. The notion of universality over countable models was first analyzed by Shelah in Theorem 1.4(3) of [Sh 87a].

Definition 2.3. (1) Let $\kappa$ be a cardinal $\geq \operatorname{LS}(\mathcal{K})$. We say $N$ is $\kappa$ universal over $M$ iff for every $M^{\prime} \in \mathcal{K}_{\kappa}$ with $M \prec \mathcal{K} M^{\prime}$ there exists a $\mathcal{K}$-embedding $g: M^{\prime} \rightarrow N$ such that $g \uparrow M=\operatorname{id}_{M}$ :

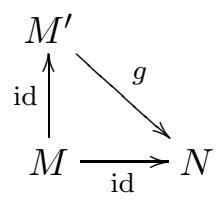

(2) We say $N$ is universal over $M$ or $N$ is a universal extension of $M$ whenever $N$ is $\|M\|$-universal over $M$.

Remark 2.4. Notice that the definition of $N$ universal over $M$ requires all extensions of $M$ of cardinality $\|M\|$ to be embeddable into $N$. Variants of this definition in the literature often involve $\|M\|<\|N\|$. We would like to emphasize that in this paper we are concentrating on the case when $\|M\|=\|N\|$. 
Examples 2.5. (1) Suppose that $T$ is a first-order complete theory that is stable in some regular $\mu$. Then every model $M$ of $T$ of cardinality $\mu$ has an elementary extension $N$ of cardinality $\mu$ which is universal over $M$. To see this define an elementary-increasing and continuous chain of models of $T$ of cardinality $\mu,\left\langle N_{i} \mid i<\mu\right\rangle$ such that $N_{i+1}$ realizes all types over $N_{i}$. Let $N=\bigcup_{i<\mu} N_{i}$. By a back-and-forth construction, one can show that $N$ is universal over $M$.

(2) Let $\mathcal{K}$ be the class of atomic models of a complete first-order theory in a countable language and $\prec_{\mathcal{K}}$ the usual notion of first-order elementary submodel. If $1 \leq \mathrm{I}\left(\aleph_{1}, \mathcal{K}\right)<2^{\aleph_{1}}$ and $2^{\aleph_{0}}<2^{\aleph_{1}}$ then for every $M \in \mathcal{K}_{\aleph_{0}}$ there exists a proper extension $N \in \mathcal{K}_{\aleph_{0}}$ universal over $M$, by Theorem 1.4(3) of [Sh 87a].

Definition 2.6. For $M^{\prime}, M \in \mathcal{K}_{\mu}$ and $\sigma$ a limit ordinal with $\sigma<\mu^{+}$, we say that $M^{\prime}$ is a $(\mu, \sigma)$-limit over $M$ iff there exists a $\prec \mathcal{K}$-increasing and continuous sequence of models $\left\langle M_{i} \in \mathcal{K}_{\mu} \mid i<\sigma\right\rangle$ such that

(1) $M=M_{0}$,

(2) $M^{\prime}=\bigcup_{i<\sigma} M_{i}$ and

(3) $M_{i+1}$ is universal over $M_{i}$.

Remark 2.7. Notice that in Definition 2.6 for $i<\sigma$ and $i$ a limit ordinal, $M_{i}$ is a $(\mu, i)$-limit model.

Definition 2.8. We say that $M^{\prime}$ is a $(\mu, \sigma)$-limit iff there is some $M \in \mathcal{K}$ such that $M^{\prime}$ is a $(\mu, \sigma)$-limit over $M$.

When $\mathcal{K}=\operatorname{Mod}(T)$ for a first-order and stable $T$ then automatically (by Theorem III.3.12 of [Shc] $)$ :

$$
\begin{aligned}
M \in \mathcal{K}_{\mu} \text { is saturated } \Longrightarrow & M \text { is }(\mu, \sigma) \text {-limit for all } \sigma<\mu^{+} \\
& \text {of cofinality } \geq \kappa(T) .
\end{aligned}
$$

The uniqueness of $(\mu, \sigma)$-limit models is an interesting problem: The statement is

(*) If $N_{\ell}$ are $\left(\mu, \sigma_{\ell}\right)$-limit models over $M$ then $N_{1} \cong_{M} N_{2}$.

A standard back and forth construction gives a uniqueness proof when $\operatorname{cf}\left(\sigma_{1}\right)=\operatorname{cf}\left(\sigma_{2}\right)$.

Problem $(*)$ for $\sigma_{1} \neq \sigma_{2}$ is important. Let's make some basic observations:

1. Suppose $\mathcal{K}=\operatorname{Mod}(T)$ and $T$ is a complete first-order theory. If $\mu$ is regular and $\sigma=\mu$ then the $(\mu, \sigma)$-limit model (if it exists) is the saturated model of $T$ of cardinality $\mu$.

2. Showing that two $(\mu, \sigma)$-limit models are isomorphic for different $\sigma \mathrm{s}$ is a central (dichotomy) property even for first-order theories: E.g. Let $T$ be a countable (for simplicity), stable, non-superstable first-order theory. Suppose $T$ is stable in $\mu$. By Harnik's theorem [Ha $T$ has a saturated model of cardinality $\mu$, in fact for $\sigma<\mu^{+}$the theory has $(\mu, \sigma)$-limit models. Since $T$ is not superstable one can modify the construction in Thm 13.1 of $\underline{\mathrm{AlGr}}$ 
to construct a $(\mu, \omega)$-limit model which is not saturated. there exists a union of an $\omega$-sequence of saturated models which is not saturated (see $\mathrm{AlGr}$ ). The union of such as sequence is not isomorphic to a $\left(\mu, \omega_{1}\right)$-limit model (that was obtained by taking a union of saturated models, see Theorem III.3.12 of [Shc] ). Thus, when $T$ is countable and stable but not superstable, the saturated model of cardinality $\mu$ is a $\left(\mu, \omega_{1}\right)$-limit but not a $(\mu, \omega)$-limit model.

We will see that under the amalgamation property, the existence of limit models follows from stability. Limit models have been applied in several contexts including Sh 394, Sh 576] and Sh 600. Even without the amalgamation property, the existence [ShVi] and uniqueness $\mathrm{Va}$ ] have been shown for categorical AECs under some mild set-theoretic assumptions.

Lately Grossberg, VanDieren and Villaveces proved uniqueness of limit models under mild model-theoretic assumptions on the AEC without assuming categoricity or working outside of ZFC (see [GVV]).

We now describe how one proves the existence of universal extensions and limit models in stable AECs. It turns out that for first-order theories stable in $\mu$ any model of cardinality $\mu$ has a universal extension of cardinality $\mu$. The result for AECs is claimed in [Sh600] without a proof.

Claim 2.9 (Claim 1.15.1 from Sh 600). Suppose $\mathcal{K}$ is an abstract elementary class with the amalgamation property. If $\mathcal{K}$ is Galois-stable in $\mu$, then for every $M \in \mathcal{K}_{\mu}$, there exists $M^{\prime} \in \mathcal{K}_{\mu}$ such that $M^{\prime}$ is universal over $M$. Moreover $M^{\prime}$ can be chosen to be a $(\mu, \sigma)$-limit over $M$ for any $\sigma<\mu^{+}$.

We provide a proof of this claim here for completeness.

As it is easy to prove:

Proposition 2.10. Suppose $\kappa \geq \operatorname{LS}(\mathcal{K}), \mathcal{K}$ has the $\kappa$-amalgamation property and $M, N \in \mathcal{K}_{\kappa}$. If $M \supsetneqq \mathcal{K} N$ and $N$ is maximal (i.e. there is no $N^{\prime} \varsubsetneqq \mathcal{K} N$ ) then $N$ is universal over $M$.

For the rest of this paper we assume:

Assumption 2.11. $\mathcal{K}$ does not have maximal models.

This is a reasonable assumption, since (in the presence of the disjoint amalgamation property) it holds automatically for categorical classes or classes with the joint embedding property.

Theorem 2.12. Let $\mathcal{K}$ be an $A E C$ which is 1 -Galois-stable in $\mu$. If $\mathcal{K}$ has the amalgamation property then for every $M \in \mathcal{K}_{\mu}$ there exists $M^{*} \nsucceq \mathcal{K} M$, universal over $M$ of cardinality $\mu$.

Proof. Define an increasing continuous chain $\left\langle M_{i}\right| i\langle\mu\rangle \subseteq \mathcal{K}_{\mu}$ such that $M_{0}:=M$ and every $p \in$ ga-S $\left(M_{i}\right)$ is realized in $M_{i+1}$. Let $M^{*}:=\bigcup_{i<\mu} M_{i}$.

We now show that $M^{*}$ is universal over $M$. Let $N \succ_{\mathcal{K}} M$ be a given model of cardinality $\mu$, we'll construct an embedding $f: N \rightarrow M^{*}$ s.t. $f\left\lceil M=\mathrm{id}_{M}\right.$. 
Fix $\left\{a_{i} \mid i<\mu\right\}$ an enumeration of $|N|-|M|$.

By induction on $i<\mu$ define $\prec_{\mathcal{K}}$-increasing and continuous chains $\left\{N_{\ell}^{i} \mid i<\mu, \ell=0,1\right\} \subseteq \mathcal{K}_{\mu}$ and $\left\{f_{i} \mid i<\mu\right\}$ such that

(1) $N_{0}^{i} \prec \mathcal{K} N_{1}^{i}$,

(2) $N_{0}^{0}=M, N_{1}^{0}=N$

(3) $a_{i} \in N_{0}^{i+1}$

(4) $f_{i}: N_{0}^{i} \rightarrow M_{i}$.

Clearly this is sufficient since $\left(\bigcup_{i<\mu} f_{i}\right) \uparrow N$ is as required. By continuity it is enough to describe the construction at successor stages.

If $a_{j} \in N_{0}^{j}$ let $N_{\ell}^{j+1}:=N_{\ell}^{j}$.

Otherwise, denote by $M_{0}^{j} \prec \mathcal{K} M_{j}$ the image of $N_{0}^{j}$ under $f_{j}$, let $g \supseteq f_{j}$ and $\bar{M}_{1}^{j} \succ_{\mathcal{K}} M_{0}^{j}$ be such that $g: N_{1}^{j} \cong \bar{M}_{1}^{j}$.

By the amalgamation property there are $M_{1}^{j}$ and $\bar{g}: \bar{M}_{1}^{j} \rightarrow M_{1}^{j}$ whose restriction is the identity over $M_{1}^{j}$ such that the diagram

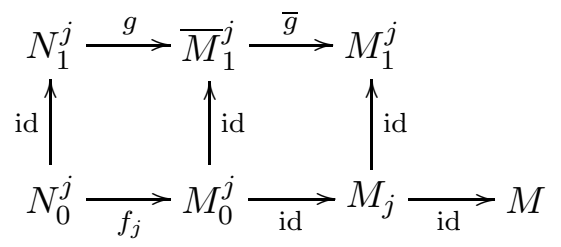

commutes.

Since $a_{j} \in N_{1}^{j}-N_{0}^{j}, g\left(a_{j}\right) \in \bar{M}_{1}^{j}-M_{0}^{j}$. We can choose $\bar{g}$ such that $\bar{g}\left(g\left(a_{j}\right)\right) \notin M_{j}$. Let $p:=\operatorname{ga-tp}\left(\bar{g}\left(g\left(a_{j}\right)\right) / M_{j}, M_{1}^{j}\right)$. By the construction of $\left\langle M_{i}\right\rangle_{i<\mu}$ there exists $b \in M_{j+1}$ realizing the type $p$. By definition of Galoistypes we have $\left(M_{j}, M_{1}^{j}, \bar{g}\left(g\left(a_{j}\right)\right)\right) E\left(M_{j}, M_{j+1}, b\right)$. Using the definition of $E$ (Definition 1.1), there are $N^{* *} \in \mathcal{K}_{\mu}$ and mappings $h_{1}, h_{2}$ such that the diagram

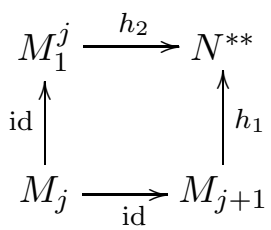

commutes and

$$
(*) \quad h_{2}\left(\bar{g}\left(g_{j}\left(a_{j}\right)\right)\right)=h_{1}(b) .
$$

We may assume that $h_{2}=\mathrm{id}_{M_{1}^{j}}$. Thus by gluing the last two diagrams together we get that the diagram

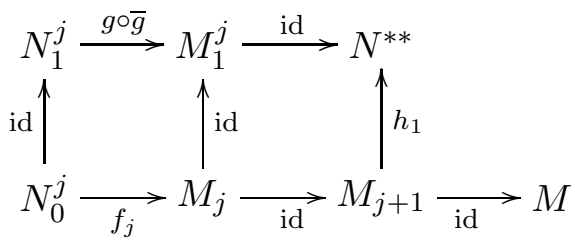


commutes. Now pick $N_{1}^{j+1} \succ_{\mathcal{K}} N_{1}^{j}$ and $h \supseteq g \circ \bar{g}$ such that $h: N_{1}^{i} \cong N^{* *}$.

So we have that

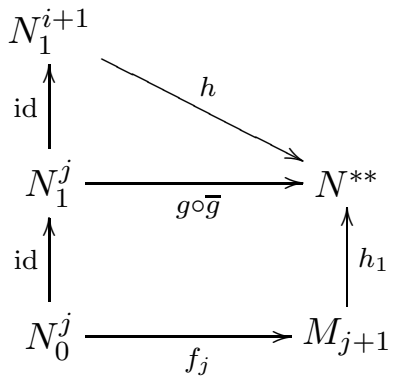

commutes. Let $N_{0}^{j+1}:=h^{-1}\left[h_{1}\left[M_{j+1}\right]\right]$.

Notice that by the above diagram, we get that $N_{0}^{j+1} \supseteq N_{0}^{j}$. Now we are ready to define the mapping $f_{i}$, let $f_{j+1}:=h_{1}^{-1} \circ\left(h \uparrow N_{0}^{j}\right)$. It is a $\mathcal{K}$ embedding that extends $f_{j}$ as required.

One can easily verify that $\bigcup_{i<\mu} f_{i}$ is an embedding of $N_{2}$ over $N_{1}$ into $M$.

The following is now immediate

Corollary 2.13. Let $\mathcal{K}$ be an $A E C$ without maximal models and suppose it is Galois-stable in $\mu$. If $\mathcal{K}$ has the amalgamation property and arbitrarily large models then for every $M \in \mathcal{K}_{\mu}$ and every $\sigma<\mu^{+}$there exists $N \in \mathcal{K}_{\mu}$ $a(\mu, \sigma)$-limit model over $M$.

Remark 2.14. In a preliminary draft of this paper we included the $\mu$ Disjoint Amalgamation Property as one of the assumptions of Theorem 2.12 We thank John Baldwin and Alexei Kolesnikov for analyzing our proof and bringing to our attention that we actually didn't use disjoint amalgamation.

\section{Tame Abstract Elementary Classes}

In AECs we view types as equivalences classes of triples (Definition 1.6) instead of sets of formulas. However, a localized equivalence relation (denoted $E_{\mu}$ ) is eventually utilized in various partial solutions to Shelah's Categoricity Conjecture (see Sh 394] and Sh 576]).

Shelah identified $E_{\mu}$ in Sh 394. Here we recall the definition.

Definition 3.1. Triples $\left(\bar{a}_{1}, M, N_{1}\right)$ and $\left(\bar{a}_{2}, M, N_{2}\right)$ are said to be $E_{\mu}$ related provided that for every $M^{\prime} \prec \mathcal{K} M$ with $M^{\prime} \in \mathcal{K}_{\leq \mu}$,

$$
\left(\bar{a}_{1}, M^{\prime}, N_{1}\right) E\left(\bar{a}_{2}, M^{\prime}, N_{2}\right) \text {. }
$$

Notice that in first order logic, the finite character of consistency implies that two types are equal if and only if they are $E_{<\omega}$-related.

Main Claim 2.3 of part II on page 288 Sh 394 Shelah ultimately proves that, under categoricity in some $\lambda>\beth_{\left(2^{\operatorname{Hanf}(\mathcal{K})}\right)+}$ and under the assumption 
that $\mathcal{K}$ has the amalgamation property, $E$-equivalence is the same as $E_{\mu}$ equivalence for 1-types over saturated models, for some $\mu<\lambda$.

We now define a context for abstract elementary classes where consistency has small character.

Definition 3.2. Let $\chi$ be a cardinal number. For $\beta>0$, we say $\mathcal{K}$ is $\chi$-tame for $\beta$-types provided that for every $M \in \mathcal{K}_{>\chi}, p \neq q \in$ ga-S ${ }^{\beta}(M)$ implies existence of $N \prec \mathcal{K} M$ of cardinality $\chi$ such that $p \uparrow N \neq q \uparrow N$. We say $\mathcal{K}$ is $\chi$-tame if it is $\chi$-tame for 1-types.

Notice that in Sh 394 Shelah defines a relative to tameness which considers only those types over saturated models. Here we are interested in types over arbitrary models.

Definition 3.3. $\mathcal{K}$ is tame iff there exists a $\chi<\operatorname{Hanf}(\mathcal{K})$ such that $\mathcal{K}$ is $\chi$-tame.

The relationship between tameness and $\chi$-tameness is not completely clear. It is plausible that the following holds:

Conjecture 3.4. Let $\mathcal{K}$ be an $A E C$. If $\mathcal{K}$ is $\chi$-tame for some $\chi$ then it is tame.

Remark 3.5. In this paper, we will actually only use that $E$-equivalence is the same as $E_{\chi}$-equivalence for types over limit models.

Notice that if $\mathcal{K}$ is a finite diagram in the sense of Sh3 (i.e. we have amalgamation not only over all models but also over all subsets of every model), then $\mathcal{K}$ is a tame AEC.

There are tame AECs with amalgamation which are not finite diagrams. In fact Leo Marcus in [Ma constructed an $L_{\omega_{1}, \omega}$-sentence which is categorical in every cardinality but does not have an uncountable sequentially homogeneous model. Recently Boris Zilber found a mathematically more natural example Zi motivated by Schanuel's Conjecture. His example is not homogeneous nor $L_{\omega_{1}, \omega}$-axiomatizable.

Shelah proves excellence (from $\mathbf{V}=\mathbf{L}$ ) for countable $L_{\omega_{1}, \omega}$-theories satisfying $I\left(\aleph_{n+1}, \mathcal{K}\right)<2^{\aleph_{n+1}}$ for all $n<\omega$ ( Sh 87a] and Sh 87b] $)$.

In GrKo] it is shown that excellence implies that $\mathcal{K}$ is $\aleph_{0}$-tame. In fact, a certain two dimensional property weaker than $\left(<\chi, \mathcal{P}^{-}(3)\right)$-AP already implies $\chi$-tameness. Even more examples of tame classes which are not finite diagrams can be found from the work of Villaveces and Zambrano (see ViZa or $\mathrm{Ba}$ ).

Let $\mathcal{K}$ be an $\mathrm{AEC}$, suppose $\kappa \geq \mathrm{LS}(\mathcal{K})$ is a strongly compact cardinal. If $\mathcal{K}$ is categorical in some $\lambda^{+}>\beth_{\left(2^{\kappa}\right)^{+}}$then by Proposition 1.13 of [MaSh] $\mathcal{K}_{\geq \kappa}$ has the amalgamation property and since Galois types can be identified with sets of formulas in $L_{\kappa, \kappa}$ (see MaSh). Thus classes satisfying the above assumptions (categoricity above the Hanf number of a strongly compact cardinal), by Makkai and Shelah's results fit into our framework of $\kappa$-tame classes. 
While we are convinced that there are examples of arbitrary levels of tameness at the time of submission of this paper we did not have any.

Question 3.6. For $\mu_{1}<\mu_{2}<\beth_{\omega_{1}}$, find an AEC which is $\mu_{2}$-tame but not $\mu_{1}$-tame.

Since the submission of our paper, some progress on this question has been made. Back in a discussion with Baldwin in Bogotá in 2001 we suggested that perhaps a non-free but almost free group of cardinality $\chi$ could be used to find an example of a class which is not $\chi$-tame. In August 2005 Baldwin informed us that he and Shelah have a work in progress for class which is not $\aleph_{1}$-tame they obtain this with groups and short exact sequences, by essentially coding the functor $\operatorname{Ext}(G, \mathbf{Z})$ and using an $\aleph_{1}$-free group of cardinality $\aleph_{1}$ which is not free and not Whitehead (see BaSh]).

\section{BOUND ON $\kappa_{\mu}^{\beta}$}

In this section we study a non-elementary relative to the first order notion of splitting and we derive bounds for an invariant that corresponds to $\kappa(T)$ under the assumption of stability and tameness.

First let us recall the notion of $\mu$-splitting introduced by Shelah in Sh 394].

Definition 4.1. A type $p \in$ ga-S $^{\beta}(N) \mu$-splits over $M \prec \mathcal{K} N$ if and only if $\|M\| \leq \mu$, there exist $N_{1}, N_{2} \in \mathcal{K}_{\leq \mu}$ and $h$, a $\mathcal{K}$-embedding such that $M \prec_{\mathcal{K}} N_{l} \prec_{\mathcal{K}} N$ for $l=1,2$ and $h: N_{1} \rightarrow N_{2}$ such that $h \uparrow M=\operatorname{id}_{M}$ and $p\left\lceil N_{2} \neq h\left(p\left\lceil N_{1}\right)\right.\right.$.

Remark 4.2. If $T$ is a first-order theory stable in $\mu$ and $M$ is saturated, then for all $N \prec M$ of cardinality $\mu$, the first order type, $\operatorname{tp}(a / M)$, does not split (in the first order sense) over $N$ iff ga-tp $(a / M)$ does not $\mu$-split over $N$.

Notice that non-splitting is monotonic: I.e. If $p \in \operatorname{ga-} \mathrm{S}(N)$ does not $\mu$ split over $M$ (for some $M \prec \mathcal{K} N$ ) then $p$ does not $\mu$-split over $M^{\prime}$ for every $M \prec \mathcal{K} M^{\prime} \prec \mathcal{K} N$ of cardinality $\mu$.

Similar to $\kappa(T)$ when $T$ is first-order the following is a natural cardinal invariant of $\mathcal{K}$ :

Definition 4.3. Let $\beta>0$. We define $\kappa_{\mu}^{\beta}(\mathcal{K})$ to be the minimal $\kappa<\mu^{+}$ such that for every $\left\langle M_{i} \in \mathcal{K}_{\mu} \mid i \leq \kappa\right\rangle$ which satisfies

(1) $\kappa=\operatorname{cf}(\kappa)<\mu^{+}$

(2) $\left\langle M_{i} \mid i \leq \kappa\right\rangle$ is $\prec \mathcal{K}$-increasing and continuous and

(3) for every $i<\kappa, M_{i+1}$ is a $(\mu, \theta)$-limit over $M_{i}$ for some $\theta<\mu^{+}$,

and for every $p \in$ ga-S $\mathrm{S}^{\beta}\left(M_{\kappa}\right)$, there exists $i<\kappa$ such that $p$ does not $\mu$-split over $M_{i}$. If no such $\kappa$ exists, we say $\kappa_{\mu}^{\beta}(\mathcal{K})=\infty$.

Another relative of $\kappa(T)$ is the following 
Definition 4.4. For $\beta>0, \bar{\kappa}_{\mu}^{\beta}(\mathcal{K})$ is the minimal cardinal $\bar{\kappa}$ such that for every $N \in \mathcal{K}_{\mu}$ and every $p \in$ ga-S $^{\beta}(N)$ there are $\lambda<\bar{\kappa}$ and $M \in \mathcal{K}_{\lambda}$ such that $p$ does not $\lambda$-split over $M$.

It is not difficult to verify that

Proposition 4.5. For $\mu$ with $\operatorname{cf}(\mu)>\bar{\kappa}_{\mu}^{\beta}(\mathcal{K})$, we have $\kappa_{\mu}^{\beta}(\mathcal{K}) \leq \bar{\kappa}_{\mu}^{\beta}(\mathcal{K})$.

While for stable first order theories (when $\beta<\omega$ ) both invariants are equal, the situation for non-elementary classes is more complicated. Already in Sh 394 it was observed that $\kappa_{\mu}^{\beta}(\mathcal{K})$ is better behaved than $\bar{\kappa}_{\mu}^{\beta}(\mathcal{K})$ when a bound for $\kappa_{\mu}^{\beta}(\mathcal{K})$ was found. A corresponding bound for $\bar{\kappa}_{\mu}^{\beta}(\mathcal{K})$ is unknown. We will defer dealing with the invariant $\bar{\kappa}_{\mu}^{\beta}(\mathcal{K})$ to a future paper.

Notice that Theorem 2.2.1 of [ShVi] states that certain categorical abstract elementary classes satisfy $\kappa_{\mu}^{1}(\mathcal{K})=\omega$, for various $\mu$. A slight modification of the argument of Claim 3.3 from Sh 394 can be used to prove a related result using the weaker assumption of Galois-stability only:

Fact 4.6. Let $\beta>0$. Suppose that $\mathcal{K}$ is $\beta$-Galois-stable in $\mu$. For every $p \in$ ga-S $^{\beta}(N)$ there exists $M \prec_{\mathcal{K}} N$ of cardinality $\mu$ such that $p$ does not $\mu$-split over $M$. Thus $\bar{\kappa}_{\mu}^{\beta}(\mathcal{K}) \leq \mu$.

For the sake of completeness an argument for Fact 4.6 is included:

Proof. Suppose $p=\operatorname{ga-tp}(\bar{a} / N, \mathfrak{C})$ with $\bar{a} \in{ }^{\beta}|\mathfrak{C}|$ and $p \mu$-splits over $M$, for every $M \prec \mathcal{K} N$ of cardinality $\mu$. We may assume that $\bar{a} \notin N$, since it is routine to check that an algebraic type never splits over its domain.

Let $\chi:=\min \left\{\chi \mid 2^{\chi}>\mu\right\}$. Notice that $\chi \leq \mu$ and $2^{<\chi} \leq \mu$.

First we will define $\left\langle M_{\alpha}, N_{\alpha, \ell} \prec N \mid \alpha<\chi, \ell=1,2\right\rangle \subseteq \mathcal{K}_{\mu}$ which will then be used to construct another sequence $\left\langle M_{\alpha}^{*} \in \mathcal{K}_{\mu} \mid \alpha \leq \chi\right\rangle$ so that

$$
\mid \text { ga-S }^{\beta}\left(M_{\chi}^{*}\right) \mid \geq 2^{\chi}>\mu \text { will witness Galois-instability in } \mu \text {. }
$$

The definition of $\left\langle M_{\alpha}, N_{\alpha, 1}, N_{\alpha, 2} \mid \alpha<\chi\right\rangle$ follows by induction on $\alpha$ and our choice of $p$. Let $M_{0} \prec M$ be any model of cardinality $\mu$.

For $\alpha=\gamma+1$, by our choice of $p$, we know $p$ must $\mu$-split over $M_{\gamma}$. Then, there are $N_{\gamma, \ell} \prec_{\mathcal{K}} M$ of cardinality $\mu$ for $\ell=1,2$ and there is $F_{\gamma}: N_{\gamma, 1} \cong_{M_{\gamma}}$ $N_{\gamma, 2}$ such that $F_{\gamma}\left(p\left\lceil N_{\gamma, 1}\right) \neq p\left\lceil N_{\gamma, 2}\right.\right.$. Pick $M_{\alpha} \prec \mathcal{K} M$ of cardinality $\mu$ containing the set $\left|N_{\gamma, 1}\right| \cup\left|N_{\gamma, 2}\right|$.

When $\alpha$ is a limit ordinal we define $M_{\alpha}:=\bigcup_{\gamma<\alpha} M_{\gamma}$.

Now we use our sequence $\left\langle M_{\alpha} \mid \alpha<\chi\right\rangle$ to define for $\alpha \leq \chi$ another sequence of models $M_{\alpha}^{*} \in \mathcal{K}_{\mu}$ and a tree of $\mathcal{K}$-embeddings $\left\langle h_{\eta} \mid \eta \in{ }^{\alpha} 2\right\rangle$ satisfying

(1) $\gamma<\alpha \Longrightarrow M_{\gamma}^{*} \prec \mathcal{K} M_{\alpha}^{*}$,

(2) for $\alpha$ limit let $M_{\alpha}^{*}=\bigcup_{\gamma<\alpha} M_{\gamma}^{*}$,

(3) $\gamma<\alpha \wedge \eta \in{ }^{\alpha} 2 \Longrightarrow h_{\eta \mid \gamma} \subseteq h_{\eta}$,

(4) $\eta \in{ }^{\alpha} 2 \Longrightarrow h_{\eta}: M_{\alpha} \rightarrow M_{\alpha}^{*}$ and

(5) $\alpha=\gamma+1 \wedge \eta \in{ }^{\alpha} 2 \Longrightarrow h_{\eta^{\wedge} 0}\left(N_{\gamma, 1}\right)=h_{\eta^{\wedge} 1}\left(N_{\gamma, 2}\right)$ 
The construction is possible by using the $\mu$-amalgamation property several times. For $\alpha=0$, define $M_{0}^{*}=M$ and $h_{\langle\rangle}:=\operatorname{id}_{M_{0}^{*}}$. When $\alpha$ is a limit ordinal we define $M_{\alpha}^{*}:=\bigcup_{\gamma<\alpha} M_{\gamma}$ and for $\eta \in{ }^{\alpha} 2, h_{\eta}:=\bigcup_{\gamma<\alpha} h_{\eta\lceil\gamma}$.

Suppose we have completed the construction for $\gamma$ and $\alpha=\gamma+1$. Given $\eta \in{ }^{\gamma} 2$, let $\bar{h}_{\eta}$ be an extension of $h_{\eta}$ to an automorphism of $\mathfrak{C}$. Notice that $\bar{h}_{\eta} \circ F_{\gamma}\left(N_{\gamma, 1}\right)=\bar{h}_{\eta}\left(N_{\gamma, 2}\right)$ by our choice of $F_{\gamma}, N_{\gamma, 1}$ and $N_{\gamma, 2}$. Let $h_{\eta^{\wedge}\langle 0\rangle}$ be some extension of $\left(\bar{h}_{\eta} \uparrow N_{\gamma, 2}\right) \circ F_{\gamma}$ to $M_{\gamma+1}$ and let $h_{\eta^{\wedge}\langle 1\rangle}$ be $\bar{h}_{\eta} \uparrow M_{\gamma+1}$. Let $M_{\gamma+1}^{*}$ be a model of cardinality $\mu$ extending $M_{\gamma}^{*}$ and containing $h_{\eta^{\wedge}\langle 0\rangle}\left(M_{\gamma+1}\right)$ and $h_{\eta^{\wedge}\langle 1\rangle}\left(M_{\gamma+1}\right)$ for each $\eta \in{ }^{\gamma} 2$.

This completes the construction and for each $\eta \in \chi_{2}$ we can fix $H_{\eta} \in$ Aut( $(\mathfrak{C})$ extending $h_{\eta}$.

Claim 4.7. $\eta \neq \nu \in \chi_{2} \Longrightarrow \operatorname{ga-tp}\left(H_{\eta}(\bar{a}) / M_{\chi}^{*}\right) \neq \operatorname{ga-tp}\left(H_{\nu}(\bar{a}) / M_{\chi}^{*}\right)$.

Proof. Let $\rho:=\eta \wedge \nu$ and suppose that $\rho^{\wedge}\langle 0\rangle\left\langle\eta\right.$ and $\rho^{\wedge}\langle 1\rangle\langle\nu$. Let $\gamma$ be the length of $\rho$. Suppose for the sake of contradiction that

$$
\operatorname{ga-tp}\left(H_{\eta}(\bar{a}) / M_{\chi}^{*}\right)=\operatorname{ga}-\operatorname{tp}\left(H_{\nu}(\bar{a}) / M_{\chi}^{*}\right) .
$$

In particular,

$$
\operatorname{ga-tp}\left(H_{\eta}(\bar{a}) / H_{\rho^{\wedge}\langle 0\rangle}\left(N_{\gamma, 1}\right)\right)=\operatorname{ga}-\operatorname{tp}\left(H_{\nu}(\bar{a}) / H_{\rho^{\wedge}\langle 1\rangle}\left(N_{\gamma, 2}\right)\right) .
$$

Referring back to our choice of $\bar{h}_{\rho}$ and $h_{\rho^{\wedge}\langle 0\rangle}$, we can apply $H_{\nu}^{-1}$ to obtain

$$
\operatorname{ga-tp}\left(H_{\nu}^{-1} \circ H_{\eta}(\bar{a}) / F_{\gamma}\left(N_{\gamma, 1}\right)=\operatorname{ga-tp}\left(\bar{a} / N_{\gamma, 2}\right)\right. \text {. }
$$

All we need to verify in order to contradict our original choices of $N_{\gamma, 1}$, $N_{\gamma, 2}$ and $F_{\gamma}$ is that for some extension of $F_{\gamma}$ to an automorphism of $\mathfrak{C}$ we have that

$$
\text { (*) } \operatorname{ga-tp}\left(F_{\gamma}(\bar{a}) / N_{\gamma, 2}\right)=\operatorname{ga}-\operatorname{tp}\left(H_{\nu}^{-1} \circ H_{\eta}(\bar{a}) / N_{\gamma, 2}\right) .
$$

Notice that by our choice of $H_{\nu}$ and $H_{\eta}$ we have that $H_{\nu}^{-1} \circ H_{\eta}\left\lceil N_{\gamma, 1}=F_{\gamma}\right.$. Thus $H_{\nu}^{-1} \circ H_{\eta} \supseteq F_{\gamma}$, so by definition of the image of a type, $F_{\gamma}\left(p\left\lceil N_{\gamma, 1}\right)=\right.$ ga-tp $\left(H_{\nu}^{-1} \circ H_{\eta}(\bar{a}) / N_{\gamma, 2}\right)$. Combining this with $(*)$ we obtain a contradiction to our choice of $N_{\gamma, 1}, N_{\gamma, 2}$ and $F_{\gamma}$ which witness the splitting of $p$ over $M_{\gamma}$.

Thus Claim 4.7 gives us that $\mid$ ga-S $\left(M_{\chi}^{*}\right) \mid \geq 2^{\chi}>\mu$ and $\mathcal{K}$ is not $\beta$-Galoisstable in $\mu$.

In Theorem 4.13 below we present an improvement of Fact 4.6 for tame AECs: In case $\mathcal{K}$ is $\beta$-stable in $\mu$ for some $\mu$ above its second Hanf number then $\kappa_{\mu}^{\beta}(\mathcal{K})$ is bounded by the second Hanf number. Notice that the bound does not depend on $\mu$.

We will need to relate Galois-stability with the failure of a certain infinitary order property in order to find a bound for $\kappa_{\mu}^{\beta}(\mathcal{K})$. The order property, defined next, is an analog of the first order definition using formulas. This order property for non-elementary classes was introduced by Shelah in Sh 394. 
Definition 4.8. $\mathcal{K}$ is said to have the $\chi$-order property provided that for every $\alpha$, there exists $\left\langle\bar{d}_{i} \mid i<\alpha\right\rangle$ and where $\bar{d}_{i} \in \chi_{\mathfrak{C}}$ such that if $i_{0}<j_{0}<\alpha$ and $i_{1}<j_{1}<\alpha$,

$(*)$ then for no $f \in A u t(\mathfrak{C})$ do we have $f\left(\bar{d}_{i_{0}}{ }^{\wedge} \bar{d}_{j_{0}}\right)=\bar{d}_{j_{1}}{ }^{\wedge} \bar{d}_{i_{1}}$.

Example 4.9. Let $T$ be a first order theory in a countable language. Set $\mathcal{K}:=\operatorname{Mod}(T)$ and $\prec \mathcal{K}$ the usual elementary submodel relation. $\mathcal{K}$ has the $\aleph_{0}$-order property iff $T$ is unstable of $T$ has dop or otop.

Remark 4.10 (Trivial monotonicity). Notice that for $\kappa_{1}<\kappa_{2}$ if a class has the $\kappa_{1}$-order property then it has the $\kappa_{2}$-order property.

Fact 4.11 (Claim 4.6.3 of [Sh 394]). We may replace the phrase every $\alpha$ in Definition 4.8 with every $\alpha<\beth_{(2 \chi+\mathrm{LS}(\mathcal{K}))}$ and get an equivalent definition.

Fact 4.12 (Claim 4.8.2 of [Sh 394]). If $\mathcal{K}$ has the $\chi$-order property and $\mu \geq$ $\chi$, then for some $M \in \mathcal{K}_{\mu}$ we have that $\mid$ ga-S $\mathrm{S}^{\chi}(M) / E_{\chi} \mid \geq \mu^{+}$. Moreover, we can conclude that $\mathcal{K}$ is not $\chi$-Galois-stable in $\mu$.

The following is a generalization of an old theorem (Theorem 4.17 in GrLe2]) of Shelah from Sh3. A special case of this theorem is Theorem 0.1 in the abstract.

Theorem 4.13. Let $\beta>0$. Suppose that $\mathcal{K}$ is $\chi$-tame for $\beta$-types. If $\mathcal{K}$ is $\beta$-stable in $\mu$ with $\beth_{(2 \chi+\mathrm{LS}(\mathcal{K}))^{+}} \leq \mu$, then $\kappa_{\mu}^{\beta}(\mathcal{K})<\beth_{(2 \chi+\mathrm{LS}(\mathcal{K}))^{+}}$.

Proof. Let $\chi^{\prime}:=\beth_{\left(2^{\chi+\mathrm{LS}(\mathcal{K})}\right)^{+}}$. Suppose that the conclusion of the theorem does not hold. Let $\left\langle M_{i} \in \mathcal{K}_{\mu} \mid i \leq \chi^{\prime}\right\rangle$ and $p \in$ ga-S $^{\beta}\left(M_{\chi^{\prime}}\right)$ witness the failure. Namely, the following hold:

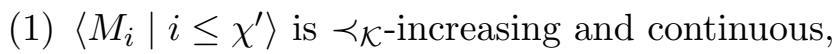

(2) for every $i<\chi^{\prime}, M_{i+1}$ is a $(\mu, \theta)$-limit over $M_{i}$ for some $\theta<\mu^{+}$and

(3) for every $i<\mu^{+}, p \mu$-splits over $M_{i}$.

For every $i<\chi^{\prime}$ let $f_{i}, N_{i}^{1}$ and $N_{i}^{2}$ witness that $p \mu$-splits over $M_{i}$. Namely,

$$
\begin{gathered}
M_{i} \prec \mathcal{K} N_{i}^{1}, N_{i}^{2} \prec \mathcal{K} M, \\
f_{i}: N_{i}^{1} \cong N_{i}^{2} \text { with } f_{i} \uparrow M_{i}=\operatorname{id}_{M_{i}} \\
\text { and } f_{i}\left(p\left\lceil N_{i}^{1}\right) \neq p\left\lceil N_{i}^{2} .\right.\right.
\end{gathered}
$$

By $\chi$-tameness, there exist models $B_{i}$ and $A_{i}:=f_{i}^{-1}\left(B_{i}\right)$ of size $<\chi$ such that

$$
f_{i}\left(p \nmid A_{i}\right) \neq p\left\lceil B_{i}\right.
$$

By renumbering our chain of models, we may assume that

(4) $A_{i}, B_{i} \subset M_{i+1}$

Since $M_{i+1}$ is a limit model over $M_{i}$, we can additionally fix

(5) $\bar{c}_{i} \in M_{i+1}$ realizing $p\left\lceil M_{i}\right.$.

For each $i<\mu$, let $\bar{d}_{i}:=A_{i}{ }^{\wedge} B_{i}{ }^{\wedge} \bar{c}_{i}$. 
Claim 4.14. $\left\langle\bar{d}_{i} \mid i<\chi^{\prime}\right\rangle$ witnesses the $\chi$-order property.

Proof. Suppose for the sake of contradiction that there exist $g \in \operatorname{Aut}(\mathfrak{C})$, $i_{0}<j_{0}<\chi^{\prime}$ and $i_{1}<j_{1}<\chi^{\prime}$ such that

$$
g\left(\bar{d}_{i_{0}} \bar{d}_{j_{0}}\right)=\bar{d}_{j_{1}} \bar{d}_{i_{1}}
$$

Notice that since $i_{0}<j_{0}<\alpha$ we have that $\bar{c}_{i_{0}} \in M_{j_{0}}$. So $f_{j_{0}}\left(\bar{c}_{i_{0}}\right)=\bar{c}_{i_{0}}$. Recall that $f_{j_{0}}\left(A_{j_{0}}\right)=B_{j_{0}}$. Thus, $f_{j_{0}}$ witnesses that

(*) $\quad$ ga-tp $\left(\bar{c}_{i_{0}}{ }^{\wedge} A_{j_{0}} / \emptyset\right)=\operatorname{ga-tp}\left(\bar{c}_{i_{0}}{ }^{\wedge} B_{j_{0}} / \emptyset\right)$.

Applying $g$ to $(*)$ we get

$$
\text { (**) } \quad \operatorname{ga-tp}\left(\bar{c}_{j_{1}}{ }^{\wedge} A_{i_{1}} / \emptyset\right)=\operatorname{ga-tp}\left(\bar{c}_{j_{1}}{ }^{\wedge} B_{i_{1}} / \emptyset\right) .
$$

Applying $f_{i_{1}}$ to the RHS of $(* *)$, we notice that

$$
(\sharp) \quad \text { ga-tp }\left(f_{i_{1}}\left(\bar{c}_{j_{1}}\right)^{\wedge} B_{i_{1}} / \emptyset\right)=\operatorname{ga}-\operatorname{tp}\left(\bar{c}_{j_{1}}{ }^{\wedge} B_{i_{1}} / \emptyset\right) \text {. }
$$

Because $i_{1}<j_{1}$, we have that $\bar{c}_{j_{1}}$ realizes $p \nmid M_{i_{1}}$. Thus, $(\sharp)$ implies

$$
f_{i_{1}}\left(p \nmid A_{i_{1}}\right)=p\left\lceil B_{i_{1}},\right.
$$

which contradicts our choice of $f_{i_{1}}, A_{i_{1}}$ and $B_{i_{1}}$.

By Claim 4.11 and Fact 4.12, we have that $\mathcal{K}$ is $\beta$-unstable in $\mu$, contradicting our hypothesis.

\section{Morley SEQUENCES}

We have now developed enough splitting machinery to derive the existence of Morley sequences for tame, Galois-stable AECs.

The following is a new Galois-theoretic notion of indiscernible sequence.

Definition 5.1. (1) $\left\langle\bar{a}_{i} \mid i<i^{*}\right\rangle$ is a Galois-indiscernible sequence over $M$ iff for every $i_{1}<\cdots<i_{n}<i^{*}$ and every $j_{1}<\cdots<j_{n}<i^{*}$, $\operatorname{ga}-\operatorname{tp}\left(\bar{a}_{i_{1}} \ldots \bar{a}_{i_{n}} / M\right)=\operatorname{ga}-\operatorname{tp}\left(\bar{a}_{j_{1}} \ldots \bar{a}_{j_{n}} / M\right)$.

(2) $\left\langle\bar{a}_{i} \mid i<i^{*}\right\rangle$ is a Galois-indiscernible sequence over $A$ iff for every $i_{1}<\cdots<i_{n}<i^{*}$ and every $j_{1}<\cdots<j_{n}<i^{*}$, there exists $M_{i}, M_{j}, M^{*} \in \mathcal{K}$ and $\prec \mathcal{K}$-mappings $f_{i}, f_{j}$ such that

(a) $A \subseteq M_{i}, M_{j}$;

(b) $f_{\ell}: M_{\ell} \rightarrow M^{*}$, for $\ell \in\{i, j\}$;

(c) $f_{i}\left(\bar{a}_{i_{0}}, \ldots, \bar{a}_{i_{n}}\right)=f_{j}\left(\bar{a}_{j_{0}}, \ldots, \bar{a}_{j_{n}}\right)$ and

(d) and $f_{i} \uparrow A=f_{j}\left\lceil A=\operatorname{id}_{A}\right.$.

Remark 5.2. This is on the surface a weaker notion of indiscernible sequence than is presented in Sh 394. However, under the amalgamation property, this definition and the definition in Sh 394 are equivalent.

Lemma 5.3 provides us with sufficient conditions to find an indiscernible sequence. 
Lemma 5.3. Let $\mu \geq \operatorname{LS}(\mathcal{K}), \kappa, \lambda$ be ordinals and $\beta$ a positive ordinal. Suppose that $\left\langle M_{i} \mid i<\lambda\right\rangle$ and $\left\langle\bar{a}_{i} \mid i<\lambda\right\rangle$ satisfy

(1) $\left\langle M_{i} \in \mathcal{K}_{\mu} \mid i<\lambda\right\rangle$ are $\prec_{\mathcal{K}}$-increasing;

(2) $M_{i+1}$ is a $(\mu, \kappa)$-limit over $M_{i}$;

(3) $\bar{a}_{i} \in{ }^{\beta} \mathrm{M}_{i+1}$;

(4) $p_{i}:=\operatorname{ga-tp}\left(\bar{a}_{i} / M_{i}, M_{i+1}\right)$ does not $\mu$-split over $M_{0}$ and

(5) for $i<j<\lambda, p_{i} \leq p_{j}$.

Then, $\left\langle\bar{a}_{i} \mid i<\lambda\right\rangle$ is a Galois-indiscernible sequence over $M_{0}$.

Definition 5.4. A sequence $\left\langle\bar{a}_{i}, M_{i} \mid i<\lambda\right\rangle$ satisfying conditions (1) - (5) of Lemma 5.3 is called a Morley sequence. It is a Morley sequence for $p \in$ ga-S $\left(\bigcup_{i<\lambda} M_{i}\right)$ if $p$ extends each ga-tp $\left(\bar{a}_{i} / M_{i}\right)$. For $A \subseteq M_{0}$, such a sequence is a Morley sequence over $A$.

Remark 5.5. Notice that our definition of Morley sequence varies from some literature. An alternative name for our sequences (suggested by John Baldwin) is a coherent non-splitting sequence.

Remark 5.6. While the statement of the lemma is similar to Shelah's first order Lemma I.2.5 in [Shc], the proof differs, since types are not sets of formulas.

Proof. We prove that for $i_{0}<\cdots<i_{n}<\lambda$ and $j_{0}<\cdots<j_{n}<\lambda$, ga-tp $\left(\bar{a}_{i_{0}}, \ldots, \bar{a}_{i_{n}} / M_{0}, M_{i_{n+1}}\right)=\operatorname{ga-tp}\left(\bar{a}_{j_{0}}, \ldots, \bar{a}_{j_{n}} / M_{0}, M_{j_{n+1}}\right)$ by induction on $n<\omega$.

$n=0$ : Let $i_{0}, j_{0}<\lambda$ be given. Condition [5, gives us

$$
\operatorname{ga-tp}\left(\bar{a}_{i_{0}} / M_{0}, M_{i_{0}+1}\right)=\operatorname{ga-tp}\left(\bar{a}_{j_{0}} / M_{0}, M_{j_{0}+1}\right) .
$$

$n>0$ : Suppose that the claim holds for all increasing sequences $\bar{i}$ and $\bar{j} \in$ $\lambda$ of length $n$. Let $i_{0}<\cdots<i_{n}<\lambda$ and $j_{0}<\cdots<j_{n}<\lambda$ be given. Without loss of generality, $i_{n} \leq j_{n}$. Define $M^{*}:=M_{1}$. From condition 2 and uniqueness of $(\mu, \omega)$-limits, we can find a $\prec \mathcal{K}$-isomorphism, $g: M_{j_{n}} \rightarrow M_{i_{n}}$ such that $g\left\lceil M_{0}=\operatorname{id}_{M_{0}}\right.$. Moreover we can extend $g$ to $g: M_{j_{n}+1} \rightarrow M_{i_{n}+1}$. Denote by $\bar{b}_{j_{l}}:=g\left(\bar{a}_{j_{l}}\right)$ for $l=0, \ldots, n$. Notice that $b_{j_{l}} \in M_{i_{n}}$ for $l<n$. Since ga-tp $\left(\bar{b}_{j_{0}}, \ldots, \bar{b}_{j_{n}} / M_{0}, M_{i_{n}+1}\right)=$ ga-tp $\left(\bar{a}_{j_{0}}, \ldots, \bar{a}_{j_{n}} / M_{0}, M_{j_{n}+1}\right)$ it suffices to prove that ga-tp $\left(\bar{b}_{j_{0}}, \ldots, \bar{b}_{j_{n}} / M_{0}, M_{i_{n}+1}\right)=\operatorname{ga-tp}\left(\bar{a}_{i_{0}}, \ldots, \bar{a}_{i_{n}} / M_{0}, M_{i_{n}+1}\right)$.

Also notice that our $\prec_{\mathcal{K}}$-mapping $g$ preserves some properties of $p_{j}$. Namely, since $p_{j}$ does not $\mu$-split over $M_{0}, g\left(p_{j}\left\lceil M_{j_{n}}\right)=p_{j} \uparrow M_{i_{n}}\right.$.

Thus, ga-tp $\left(\bar{b}_{j_{n}} / M_{i_{n}}, M_{i_{n}+1}\right)=\operatorname{ga-} \operatorname{tp}\left(\bar{a}_{j_{n}} / M_{i_{n}}, M_{i_{n}+1}\right)$. In particular we have that ga-tp $\left(\bar{b}_{j_{n}} / M_{i_{n}}, M_{i_{n}+1}\right)$ does not $\mu$-split over $M_{0}$.

By the induction hypothesis

$$
\operatorname{ga-tp}\left(\bar{b}_{j_{0}}, \ldots, \bar{b}_{j_{n-1}} / M_{0}, M_{i_{n}}\right)=\operatorname{ga-tp}\left(\bar{a}_{i_{0}}, \ldots, \bar{a}_{i_{n-1}} / M_{0}, M_{i_{n}}\right) .
$$

Thus we can find $h_{i}: M_{i_{n}+1} \rightarrow M^{*}$ and $h_{j}: M_{i_{n}+1} \rightarrow M^{*}$ such that $h_{i}\left(\bar{a}_{i_{0}}, \ldots, \bar{a}_{i_{n-1}}\right)=h_{j}\left(\bar{b}_{j_{0}}, \ldots, \bar{b}_{j_{n-1}}\right)$. Let us abbreviate $\bar{b}_{j_{0}}, \ldots, \bar{b}_{j_{n-1}}$ by $\bar{b}_{\bar{j}}$. Similarly we will write $\bar{a}_{\bar{i}}$ for $\bar{a}_{i_{0}}, \ldots, \bar{a}_{i_{n-1}}$. 
By appealing to condition 4 we derive several equalities that will be useful in the latter portion of the proof. Since $p_{j}$ does not $\mu$-split over $M_{0}$, we have that $p_{j}\left\lceil h_{j}\left(M_{i_{n}}\right)=h_{j}\left(p_{j}\left\lceil M_{i_{n}}\right)\right.\right.$, rewritten as

$(*) \quad \operatorname{ga-tp}\left(\bar{b}_{j_{n}} / h_{j}\left(M_{i_{n}}\right), M_{i_{n}+1}\right)=\operatorname{ga-tp}\left(h_{j}\left(\bar{b}_{j_{n}}\right) / h_{j}\left(M_{i_{n}}\right), M^{*}\right)$.

Similarly as $p_{i}$ does not $\mu$-split over $M_{0}$, we get

$p_{i} \uparrow h_{j}\left(M_{i_{n}}\right)=h_{j}\left(p_{i} \uparrow M_{i_{n}}\right)$ and $p_{i} \uparrow h_{i}\left(M_{i_{n}}\right)=h_{i}\left(p_{i} \uparrow M_{i_{n}}\right)$. These equalities translate to

$(* *)_{j} \quad \operatorname{ga}-\operatorname{tp}\left(\bar{a}_{i_{n}} / h_{j}\left(M_{i_{n}}\right), M_{i_{n}+1}\right)=\operatorname{ga-tp}\left(h_{j}\left(\bar{a}_{i_{n}}\right) / h_{j}\left(M_{i_{n}}\right), M^{*}\right)$ and

$(* *)_{i} \quad$ ga-tp $\left(\bar{a}_{i_{n}} / h_{i}\left(M_{i_{n}}\right), M_{i_{n}+1}\right)=$ ga-tp $\left(h_{i}\left(\bar{a}_{i_{n}}\right) / h_{i}\left(M_{i_{n}}\right), M^{*}\right)$, respectively.

Finally, from condition [5, notice that

$$
(* * *) \quad \text { ga-tp }\left(\bar{a}_{i_{n}} / M_{i_{n}}, M_{i_{n}+1}\right)=\operatorname{ga-tp}\left(\bar{b}_{j_{n}} / M_{i_{n}}, M_{i_{n}+1}\right) .
$$

Applying $h_{j}$ to $(* * *)$ yields

(†) $\quad \operatorname{ga}-\operatorname{tp}\left(h_{j}\left(\bar{b}_{j_{n}}\right) / h_{j}\left(M_{i_{n}}\right), M^{*}\right)=\operatorname{ga-tp}\left(h_{j}\left(\bar{a}_{i_{n}}\right) / h_{j}\left(M_{i_{n}}\right), M^{*}\right)$.

Since $h_{i}\left(\bar{a}_{\bar{i}}\right)=h_{j}\left(\bar{b}_{\bar{j}}\right) \in h_{j}\left(M_{i_{n}}\right)$, we can draw from $(\dagger)$ the following:

(1) $\operatorname{ga-tp}\left(h_{j}\left(\bar{b}_{j_{n}}\right)^{\wedge} h_{j}\left(\bar{b}_{\bar{j}}\right) / M_{0}, M^{*}\right)=\operatorname{ga-tp}\left(h_{j}\left(\bar{a}_{j_{n}}\right)^{\wedge} h_{i}\left(\bar{a}_{\bar{i}}\right) / M_{0}, M^{*}\right)$.

Similarly, equality $(* *)_{i}$ allows us to see

(2) $\quad \operatorname{ga}-\operatorname{tp}\left(\bar{a}_{i_{n}}{ }^{\wedge} h_{i}\left(\bar{a}_{\bar{i}}\right) / M_{0}, M^{*}\right)=\operatorname{ga}-\operatorname{tp}\left(h_{i}\left(\bar{a}_{i_{n}}\right)^{\wedge} h_{i}\left(\bar{a}_{\bar{i}}\right) / M_{0}, M^{*}\right)$.

Since ga-tp $\left(h_{j}\left(\bar{a}_{i_{n}}\right) / h_{j}\left(M_{i_{n}}\right), M^{*}\right)=\operatorname{ga-tp}\left(\bar{a}_{i_{n}} / h_{j}\left(M_{i_{n}}\right), M_{i_{n}+1}\right)$ (equality $\left.\left.(* *)_{j}\right)\right)$ and $h_{i}\left(\bar{a}_{\bar{i}}\right)=h_{j}\left(\bar{b}_{\bar{j}}\right) \in h_{j}\left(M_{i_{n}}\right)$, we get that

(3) $\operatorname{ga-tp}\left(h_{j}\left(\bar{a}_{i_{n}}\right)^{\wedge} h_{i}\left(\bar{a}_{\bar{i}}\right) / M_{0}, M^{*}\right)=\operatorname{ga-tp}\left(\bar{a}_{i_{n}}{ }^{\wedge} h_{i}\left(\bar{a}_{\bar{i}}\right) / M_{0}, M^{*}\right)$.

Combining equalities (1), (2) and (3), we get

(††) ga-tp $\left(h_{i}\left(\bar{a}_{\bar{i}}\right)^{\wedge} h_{i}\left(\bar{a}_{i_{n}}\right) / M_{0}, M^{*}\right)=\operatorname{ga-tp}\left(h_{j}\left(\bar{b}_{\bar{j}}\right)^{\wedge} h_{j}\left(\bar{b}_{j_{n}}\right) / M_{0}, M^{*}\right)$.

Recall that $h_{i} \uparrow M_{0}=h_{j} \uparrow M_{0}=\operatorname{id}_{M_{0}}$. Thus ( $\left.\dagger \dagger\right)$, witnesses that

$$
\operatorname{ga-tp}\left(\bar{a}_{i_{0}}, \ldots, \bar{a}_{i_{n}} / M_{0}, M_{i_{n}+1}\right)=\operatorname{ga-tp}\left(\bar{b}_{j_{0}}, \ldots, \bar{b}_{j_{n}} / M_{0}, M_{i_{n}+1}\right) \text {. }
$$

Theorem 5.7. Fix $\beta>0$. Suppose $\mathcal{K}$ is $\chi$-tame for $\beta$-types with $\chi<$ $\beth_{\left(2^{\text {Hanf }(\mathcal{K})}\right)^{+}}$. Suppose $\mu \geq \beth_{\left(2^{\text {Hanf }(\mathcal{K})}\right)^{+}}$. Let $M \in \mathcal{K}_{>\mu}, A \subset M$ and $I \subseteq{ }^{\beta} \mathrm{M}$ be given such that $|I| \geq \mu^{+}>|A|$. If $\mathcal{K}$ is Galois $\beta$-stable in $\mu$, then there exists $J \subset I$ of cardinality $\mu^{+}$, Galois indiscernible over $A$. Moreover $J$ can be chosen to be a Morley sequence over $A$.

Proof. Fix $\kappa=\kappa_{\mu}^{\beta}$. By Theorem 4.13] $\kappa<\beth_{\left(2^{\text {Hanf }(\mathcal{K})}\right)^{+}}$. Let $\left\{\bar{a}_{i} \in I \mid i<\right.$ $\left.\mu^{+}\right\}$be given. Define $\left\langle M_{i} \in K_{\mu} \mid i<\mu^{+}\right\rangle, \prec_{\mathcal{K}}$-increasing and continuous, satisfying

(1) $A \subseteq\left|M_{0}\right|$

(2) $M_{i+1}$ is a $(\mu, \kappa)$-limit over $M_{i}$

(3) $\bar{a}_{i} \in M_{i+1}$ 
Let $p_{i}:=\operatorname{ga-} \operatorname{tp}\left(\bar{a}_{i} / M_{i}, M_{i+1}\right)$ for every $i<\mu^{+}$. Define $f: S_{\kappa}^{\mu^{+}} \rightarrow \mu^{+}$by

$$
f(i):=\min \left\{j<\mu^{+} \mid p_{i} \text { does not } \mu \text {-split over } M_{j}\right\} .
$$

By our choice of $\kappa, f$ is regressive. Thus by Fodor's Lemma, there are a stationary set $S \subseteq S_{\kappa}^{\mu^{+}}$and $j_{0}<\mu^{+}$such that for every $i \in S$,

(†) $p_{i}$ does not $\mu$-split over $M_{j_{0}}$.

By stability and the pigeon-hole principle there exists $p^{*} \in$ ga-S $\left(M_{j_{0}}\right)$ and $S^{*} \subseteq S$ of cardinality $\mu^{+}$such that for every $i \in S^{*}, p^{*}=p_{i} \uparrow M_{j_{0}}$. Let $M^{*}:=M_{j_{0}}$. Enumerate and $\left\langle M_{j_{i}} \mid i \in S^{*}\right\rangle$. Let $M^{* *}:=M_{j_{1}}$ in this enumeration. Again, by stability we can find $S^{* *} \subset S^{*}$ of cardinality $\mu^{+}$ such that for every $i \in S^{* *}, p^{* *}=p_{i}\left\lceil M^{*}\right.$. Notice that $M^{*}, M^{* *} \prec \mathcal{K} M_{i}$ for every $i \in S^{* *}$.

Subclaim 5.8. For $i<j \in S^{* *}, p_{i}=p_{j} \uparrow M_{i}$.

Proof. Let $0<i<j \in S^{* *}$ be given. Since $M_{i+1}$ and $M_{j+1}$ are $(\mu, \kappa)$ limits over $M_{i}$, there exists an isomorphism $g: M_{j+1} \rightarrow M_{i+1}$ such that $g \uparrow M_{i}=\operatorname{id}_{M_{i}}$. Let $\bar{b}_{j}:=g\left(\bar{a}_{j}\right)$. Since the type $p_{j}$ does not $\mu$-split over $M^{*}, g$ cannot witness the splitting. Therefore, it must be the case that ga-tp $\left(\bar{b}_{j} / M_{i}, M_{i+1}\right)=p_{j} \uparrow M_{i}$. Then, it suffices to show that $p_{i}=$ ga-tp $\left(\bar{b}_{j} / M_{i}, M_{i+1}\right)$.

Since $p_{i} \uparrow M^{*}=p_{j} \uparrow M^{*}$, we can find $\prec_{\mathcal{K}}$-mappings witnessing the equality. Furthermore since $M^{* *}$ is universal over $M^{*}$, we can find $h_{l}$ : $M_{l+1} \rightarrow M^{* *}$ such that $h_{l}\left\lceil M^{*}=\operatorname{id}_{M^{*}}\right.$ for $l=i, j$ and $h_{i}\left(\bar{a}_{i}\right)=h_{j}\left(\bar{b}_{j}\right)$.

We will use $(\dagger)$ to derive several inequalities. Consider the following possible witness to splitting. Let $N_{1}:=M_{i}$ and $N_{2}:=h_{i}\left(M_{i}\right)$. Since $p_{i}$ does not $\mu$-split over $M^{*}$, we have that $p_{i}\left\lceil N_{2}=h_{i}\left(p_{i}\left\lceil N_{1}\right)\right.\right.$, rewritten as

$$
\text { (*) ga-tp }\left(\bar{a}_{i} / h_{i}\left(M_{i}\right), M_{i+1}\right)=\operatorname{ga-tp}\left(h_{i}\left(\bar{a}_{i}\right) / h_{i}\left(M_{i}\right), M^{* *}\right) .
$$

Similarly we can conclude that

$$
(* *) \quad \operatorname{ga-tp}\left(\bar{b}_{j} / h_{j}\left(M_{i}\right), M_{i+1}\right)=\operatorname{ga-tp}\left(h_{j}\left(\bar{b}_{j}\right) / h_{j}\left(M_{i}\right), M^{* *}\right) .
$$

By choice of $S^{* *}$, we know that

$$
(* * *) \quad \operatorname{ga}-\operatorname{tp}\left(\bar{b}_{j} / M^{* *}\right)=\operatorname{ga}-\operatorname{tp}\left(\bar{a}_{i} / M^{* *}\right) .
$$

Now let us consider another potential witness of splitting. $N_{1}^{*}:=h_{i}\left(M_{i}\right)$ and $N_{2}^{*}:=h_{j}\left(M_{i}\right)$ with $H^{*}:=h_{j} \circ h_{i}^{-1}: N_{1}^{*} \rightarrow N_{2}^{*}$. Since $p_{j}\left\lceil M_{i}\right.$ does not $\mu$-split over $M_{0}, p_{j}\left\lceil N_{2}^{*}=H^{*}\left(p_{j}\left\lceil N_{1}^{*}\right)\right.\right.$. Thus by $(* *)$ we have

$$
(\sharp) \quad H^{*}\left(p_{j} \uparrow N_{1}^{*}\right)=\operatorname{ga-tp}\left(h_{j}\left(\bar{b}_{j}\right) / h_{j}\left(M_{i}\right), M^{* *}\right) .
$$

Now let us translate $H^{*}\left(p_{j}\left\lceil N_{1}^{*}\right)\right.$. By monotonicity and $(* * *)$, we have that $p_{j}\left\lceil N_{1}^{*}=\operatorname{ga-tp}\left(\bar{b}_{j} / h_{i}\left(M_{i}\right), M_{i+1}\right)=\operatorname{ga-tp}\left(\bar{a}_{i} / h_{i}\left(M_{i}\right), M_{i+1}\right)\right.$. We can then conclude by $(*)$ that $p_{j} \uparrow N_{1}^{*}=\operatorname{ga-tp}\left(h_{i}\left(\bar{a}_{i}\right) / h_{i}\left(M_{i}\right), M_{i+1}\right)$. Applying $H^{*}$ to this equality yields

$$
(\sharp \sharp) \quad H^{*}\left(p_{j} \uparrow N_{1}^{*}\right)=\operatorname{ga-tp}\left(h_{j}\left(\bar{a}_{i}\right) / h_{j}\left(M_{i}\right), M^{* *)} .\right.
$$


By combining the equalities from $(\sharp)$ and $(\sharp \sharp)$ and applying $h_{j}^{-1}$ we get that

$$
\text { ga-tp }\left(\bar{b}_{j} / M_{i}, M_{i+1}\right)=\operatorname{ga-tp}\left(\bar{a}_{i} / M_{i}, M_{i+1}\right) .
$$

Notice that by Subclaim 5.8 and our choice of $S^{* *},\left\langle M_{i} \mid i \in S^{* *}\right\rangle$ and $J:=\left\langle\bar{a}_{i} \mid i \in S^{* *}\right\rangle$ satisfy the conditions of Lemma [5.3 Applying Lemma 5.3. we get that $\left\langle\bar{a}_{i} \mid i \in S^{* *}\right\rangle$ is a Morley sequence over $M^{*}$. In particular, since $A \subset M^{*}$, we have that $\left\langle\bar{a}_{i} \mid i \in S^{* *}\right\rangle$ is a Morley sequence over $A$.

Remark 5.9. Our effort to prove the results of this section in summer 2001 lead us to the notion of tameness as defined in this paper. A preliminary version of this paper containing the results of this section was posted by us on the web already in July 27th, 2001 with the title Morley Sequences in AECs.

\section{Towards a Stability Spectrum Theorem}

In this section we begin work towards a stability spectrum theorem for tame classes. Other partial results in this direction appear in BaKuVa.

Theorem 6.1 (Extension property for non- $\mu$-splitting types). Let $\mathcal{K}$ be an AEC. Suppose $N \prec_{\mathcal{K}} M^{\prime} \prec_{\mathcal{K}} M$ are such that $M^{\prime}$ is universal over $N$ and all three models have cardinality $\mu$. Then for every $p^{\prime} \in$ ga-S $\left(M^{\prime}\right)$ which does not $\mu$-split over $N$, there exists $p \in$ ga-S $(M)$ extending $p^{\prime}$ such that $p$ does not $\mu$-split over $N$.

Proof. Let $N, M^{\prime}, M$ be as in the statement of the theorem. Suppose $p=$ ga-tp $\left(a^{\prime} / M^{\prime}\right)$ does not $\mu$-split over $N$. Since $M^{\prime}$ is universal over $N$, there exists $f: M^{\prime} \rightarrow M$ with $f \uparrow N=\operatorname{id}_{N}$. Notice that by monotonicity of non$\mu$-splitting, we have that ga-tp $\left(a^{\prime} / f(M)\right)$ does not $\mu$-split over $N$. Then, by invariance we have that also

$$
\text { (*) } \quad \text { ga-tp }\left(f\left(a^{\prime}\right) / M\right) \text { does not } \mu \text {-split over } N \text {. }
$$

By one more appeal to non-splitting, we can conclude that ga-tp $\left(f\left(a^{\prime}\right) / M^{\prime}\right)=$ ga-tp $\left(a^{\prime} / M^{\prime}\right)$. Otherwise these two types would contradict $(*)$.

Theorem 6.2 (Uniqueness of non-splitting extensions). Let $\mathcal{K}$ be $\chi$-tame abstract elementary class and $\mu$ a cardinal with $\mu \geq \chi$. Let $M \in \mathcal{K}_{>\mu}$ and $M^{\prime}, N \in \mathcal{K}_{\mu}$ with $N \prec_{\mathcal{K}} M^{\prime} \prec_{\mathcal{K}} M$. If $M^{\prime}$ is universal over $N$, then for every $p^{\prime} \in$ ga-S $\left(M^{\prime}\right)$ which does not $\mu$-split over $N$, if there are $q, p \in \operatorname{ga-S}(M)$ such that $q$ and $p$ both extend $p^{\prime}$ and do not $\mu$-split over $N$, then $p=q$.

Proof. Suppose for the sake of contradiction that there exists $q \neq p \in$ ga-S $(M)$ extending $p^{\prime} \in$ ga-S $\left(M^{\prime}\right)$ such that both $p$ and $q$ do not $\mu$-split over $N$. Let $a, b$ be such that $p=\operatorname{ga-tp}(a / M)$ and $q=$ ga-tp $(b / M)$. By tameness there exists $M^{*} \in \mathcal{K}_{\mu}$ such that $M^{\prime} \prec_{\mathcal{K}} M^{*} \prec_{\mathcal{K}} M$ and $p\left\lceil M^{*} \neq q \uparrow M^{*}\right.$. 


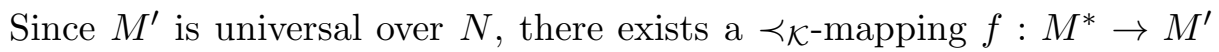
with $f \uparrow N=\operatorname{id}_{N}$. Since $p$ and $q$ do not $\mu$-split over $N$ we have

$$
\begin{gathered}
(*)_{a} \quad \operatorname{ga-tp}\left(a / f\left(M^{*}\right)\right)=\operatorname{ga-tp}\left(f(a) / f\left(M^{*}\right)\right) \text { and } \\
(*)_{b} \quad \operatorname{ga-tp}\left(b / f\left(M^{*}\right)\right)=\operatorname{ga-tp}\left(f(b) / f\left(M^{*}\right)\right) .
\end{gathered}
$$

On the other hand, since $p \uparrow M^{*} \neq q \uparrow M^{*}$, we have that their images are also not equal

$$
\text { (*) } \quad \text { ga-tp }\left(f(a) / f\left(M^{*}\right)\right) \neq \operatorname{ga-tp}\left(f(b) / f\left(M^{*}\right)\right) .
$$

Combining $(*)_{a},(*)_{b}$ and $(*)$, we get

$$
\text { ga-tp }\left(a / f\left(M^{*}\right)\right) \neq \operatorname{ga-tp}\left(b / f\left(M^{*}\right)\right) .
$$

Since $f\left(M^{*}\right) \prec \mathcal{K} M^{\prime}$, this inequality witnesses that

$$
\operatorname{ga-tp}\left(a / M^{\prime}\right) \neq \operatorname{ga-tp}\left(b / M^{\prime}\right),
$$

contradicting our choice of $p$ and $q$ both extending $p^{\prime}$.

Corollary 6.3. Let $\mathcal{K}$ be $\chi$-tame abstract elementary class and let $\mu$ be a cardinal with $\mu>\chi$. Let $M \in \mathcal{K}_{>\mu}, N \prec_{\mathcal{K}} M$ with $M$ universal over $N$. If $\mathcal{K}$ is Galois-stable in $\mu$ then

$$
\mid\{p \in \operatorname{ga-S}(M): p \text { does not } \mu \text {-split over } N\} \mid \leq \mu .
$$

Proof. Since $M$ is universal over $N$, there is a $M^{\prime} \in \mathcal{K}_{\mu}$ with $N \prec \mathcal{K} M^{\prime} \prec_{\mathcal{K}}$ $M$ and $M^{\prime}$ universal over $N$. By $\mu$-stability there are only $\mu$-many types over $M^{\prime}$ and by Theorem 6.2 each type over $N$ has at most one non- $\mu$-splitting extension to $M$.

Corollary 6.4 (Partial stability spectrum). Suppose that $\mathcal{K}$ is a tame $a b$ stract elementary class satisfying the amalgamation property. If $\mathcal{K}$ is Galoisstable in some $\mu>\operatorname{Hanf}(\mathcal{K})$, then $\mathcal{K}$ is stable in every $\kappa$ with $\kappa^{\mu}=\kappa$.

Proof. Let $\kappa=\kappa^{\mu}$. Consider $M \in \mathcal{K}_{\kappa}$ and let $M^{\prime}$ be a $\mu$-saturated extension of $M$. Such an extension exists by stability in $\mu$ and our cardinal arithmetic assumption. Suppose that ga-tp $(a / M, \mathfrak{C}) \neq$ ga-tp $(b / M, \mathfrak{C})$, then surely ga-tp $\left(a / M^{\prime}, \mathfrak{C}\right) \neq$ ga-tp $\left(b / M^{\prime}, \mathfrak{C}\right)$. So the number of Galois-types over $M$ is $\leq$ the number of Galois-types over $M^{\prime}$.

Thus we may assume that $M$ is $\mu$-saturated. Again by our assumption

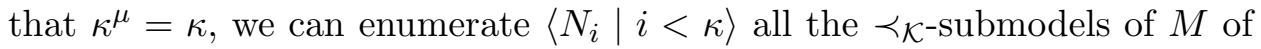
cardinality $\mu$. Since $M$ is $\mu$-saturated, for each one of the $N_{i}$ there is $M_{i}^{\prime}$ of cardinality $\mu$ such that $N_{i} \prec_{\mathcal{K}} M_{i}^{\prime} \prec_{\mathcal{K}} M$ with $M_{i}^{\prime}$ universal over $N_{i}$. By Fact 4.6] for every $p \in$ ga-S $(M)$, there exists $N_{i} \prec \mathcal{K} M$ of cardinality $\mu$ such that $p$ does not $\mu$-split over $N_{i}$. Thus

$$
\mid \text { ga-S }(M)|=| \bigcup_{i<\kappa}\left\{p \in \text { ga-S }(M) \mid p \text { does not } \mu \text {-split over } N_{i}\right\} \mid \text {. }
$$


By monotonicity of non-splitting we have that for each $p \in$ ga-S $(M)$ that does not $\mu$-split over $N_{i}$, also $p\left\lceil M_{i}^{\prime}\right.$ does not $\mu$-split over $N_{i}$. By Corollary 6.3. for each $i<\kappa$,

$$
\begin{gathered}
\quad \mid\left\{p \in \text { ga- } \mathrm{S}(M) \mid p \text { does not } \mu \text {-split over } N_{i}\right\} \mid \\
\leq \mid\left\{p \in \text { ga- } \mathrm{S}\left(M_{i}^{\prime}\right) \mid p \text { does not } \mu \text {-split over } N_{i}\right\} \mid .
\end{gathered}
$$

Now by stability in $\mu$ and our assumption that $\kappa^{\mu}=\kappa$, we can conclude that $|\operatorname{ga}-\mathrm{S}(M)| \leq \kappa$.

\section{EXERCISE ON Dividing}

With the existence of Morley sequences a natural extension is to study the following dependence relation to determine whether or not it satisfies properties such as transitivity, symmetry or extension. Here we derive the existence property for types over saturated models.

Definition 7.1. Let $p \in$ ga-S $(M)$ and $N \prec \mathcal{K} M$. We say that $p$ divides over $N$ iff there are $a \in|M| \backslash|N|$ and a Morley sequence, $\left\{a_{n} \mid n<\omega\right\}$ for the ga-tp $(a / N, M)$ such that for some collection $\left\{f_{n} \in A u t_{M} \mathfrak{C} \mid n<\omega\right\}$ with $f_{n}(a)=a_{n}$ we have

$$
\left\{f_{n}(p) \mid n<\omega\right\} \text { is inconsistent. }
$$

I.e. there is no common realization to the above mentioned $\omega$-many types.

Theorem 7.2 (Existence). Suppose that $\mathcal{K}$ is Galois-stable in $\mu$ and $\chi$-tame for some $\chi<\mu$. For every $\mu$-model homogeneous model $M \in \mathcal{K}_{\geq \mu}$ and ever $p \in \operatorname{ga-} \mathrm{S}(M)$, there exists $N \prec \mathcal{K} M$ of cardinality $\mu$ such that $p$ does not divide over $N$.

Proof. Suppose that $p$ and $M$ form a counter-example. We will find $N_{i}, N_{i}^{1}, N_{i}^{2}$ and $h_{i}$ for $i<\mu$ satisfying:

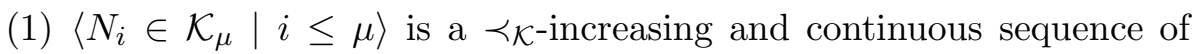
models;

(2) $N_{i} \prec \mathcal{K} N_{i}^{l} \prec \mathcal{K} N_{i+1}$ for $i<\mu$ and $l=1,2$;

(3) for $i<\mu, h_{i}: N_{i}^{1} \cong N_{i}^{2}$ and $h_{i}\left\lceil N_{i}=\operatorname{id}_{N_{i}}\right.$ and

(4) $p \nmid N_{i}^{2} \neq h_{i}\left(p\left\lceil N_{i}^{1}\right)\right.$.

Suppose that $N_{i}$ has been defined. Since $p$ divides over every substructure of cardinality $\mu$, we may find $\bar{a},\left\{\bar{a}_{n} \mid n<\omega\right\}$ and $\left\{f_{n} \mid n<\omega\right\}$ witnessing that $p$ divides over $N_{i}$. Namely, we have that $\left\{f_{n}(p) \mid n<\omega\right\}$ is inconsistent. Let $n<\omega$ be such that $f_{0}(p) \neq f_{n}(p)$. Then $p \neq f_{0}^{-1} \circ f_{n}(p)$. By $\chi-$ tameness, we can find $N^{*} \prec \mathcal{K} M$ of cardinality $\mu$ containing $N$ such that $p\left\lceil N^{*} \neq\left(f_{0}^{-1} \circ f_{n}(p)\right) \uparrow N^{*}\right.$. WLOG $f_{0}^{-1} \circ f_{n} \in A u t_{N} N^{*}$.

Let $h_{i}:=f_{0}^{-1} \circ f_{n}, N_{i}^{1}:=N^{*}$ and $N_{i}^{2}:=N^{*}$. Choose $N_{i+1} \prec \mathcal{K} M$ to be an extension of $N^{*}$ of cardinality $\mu$. 
Now we can use $\left\langle N_{i}, N_{i}^{1}, N_{i}^{2}, h_{i} \mid i<\mu\right\rangle$ to contradict Galois-stability in $\mu$ by repeating the argument from the proof of Fact 4.6. (starting at the second paragraph) we construct an increasing chain of models and a tree of $\mathcal{K}$-embedding which by Claim 4.7 gives many Galois-types contradicting stability in $\mu$.

Remark 7.3. In a previous version of this paper, we stated for the existence property for arbitrary models $M \in \mathcal{K}_{\geq \mu}$. We do not know how to remove the assumption of $\mu$-model homogeneity and we suspect that this is not possible with only the assumption of $\mu$-stability and tameness.

\section{REFERENCES}

[AlGr] M. Albert and R. Grossberg, Rich models, Journal of Symbolic Logic, 55, (1990) 1292-1298.

[Ba] John Baldwin. The Hrushovski construction and quasiminimal excellent classes. In preparation.

$[\mathrm{BaKuVa}]$ J. Baldwin, D. Kueker and M. VanDieren, Upward Stability Transfer Theorem for Tame Abstract Elementary Classes, (8 pages), Preprint available

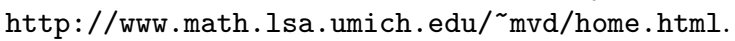

[BaSh] John Baldwin and S. Shelah. Examples of non-locality. in preparation.

[Gr1] R. Grossberg, Classification theory for non-elementary classes, Logic and Algebra, ed. Yi Zhang, Contemporary Mathematics, 302, (2002) AMS, pp. 165-204.

[Gr2] R. Grossberg, A Course in Model Theory, Book in Preparation, Available at http://www . math.cmu.edu/r̃ami/home.html.

[GrLe1] R. Grossberg and O. Lessmann, The local order property in non elementary classes, Arch Math Logic 39 (2000) 6, 439-457.

[GrLe2] R. Grossberg and O. Lessmann, Shelah's stability spectrum and homogeneity spectrum in finite diagrams, Archive for Mathematical Logic, 41, (2002) 1, 1-31.

[GrKo] R. Grossberg and A. Kolesnikov, Excellent abstract elementary classes are tame, (12 pages), preprint.

[GrVa1] R. Grossberg and M. VanDieren, Categoricity in Tame Abstract Elementary Classes, (23 pages), Preprint available at http://www .math.lsa.umich.edu/ mvd/home.html.

[GrVa2] R. Grossberg and M. VanDieren, Categoricity from one successor cardinal in Tame Abstract Elementary Classes, (17 pages), Preprint available at http://www.math.lsa.umich.edu/ ${ }^{\mathrm{mvd}}$ /home.html.

[GVV] Rami Grossberg, Monica VanDieren and Andrés Villaveces, Limit Models in Classes with Amalgamation, (18 pages), Preprint available at

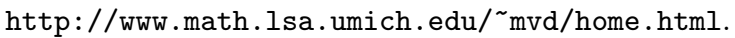

[Ha] V. Harnik, On existence of saturated models of stable theories, Proc. of AMS, 52, 361-367, 1975.

[Ma] L. Marcus, A prime minimal model with an infinite set of indiscernibles, Israel Journal of Mathematics, 11, (1972), 180-183.

[MaSh] Michael Makkai and Saharon Shelah. Categoricity of theories in $L_{\kappa \omega}$, with $\kappa$ a compact cardinal. Annals of Pure and Applied Logic, 47:41-97, 1990.

[Shc] S. Shelah, Classification Theory and the Number of Non-isomorphic Models $2^{\text {nd }}$ edition, North Holland Amsterdam, 1990.

[Sh3] S. Shelah, Finite diagrams stable in power, Ann. Math. Logic, 2, 69-118, 1970/1971.

[Sh 87a] S. Shelah, Classification theory for nonelementary classes, I, The number of uncountable models of $\psi \in L_{\omega_{1}, \omega}$, Part A, Israel J. Math., 46:212-240, 1983. 
[Sh 87b] S. Shelah, Classification theory for nonelementary classes, I, The number of uncountable models of $\psi \in L_{\omega_{1}, \omega}$, Part B, Israel J. Math., 46:241-273, 1983.

[Sh 88] S. Shelah, Classification of nonelementary classes, II, Abstract Elementary Classes. In Classification Theory (Chicago IL 1985), volume 1292 of Lecture Notes in Mathematics, pages 419-497. Springer, BSerlin, 1987. Proceedings of the USA-Israel Conference on Classification Theory, Chicago, December 1985; ed. Baldwin, J.T.

[Sh 300] S. Shelah, Universal Classes. In Classification Theory of Lecture Notes in Mathematics, 1292, 264-418. Springer-Berlin, 1987.

[Sh 394] S. Shelah, Categoricity of abstract classes with amalgamation, Annals of Pure and Applied Logic, 98(1-3), pages 141-187, 1999.

[Sh 576] S. Shelah, Categoricity of an abstract elementary class in two successive cardinals, Israel J. of Math, 126, (2001), 29-128.

[Sh 600] S. Shelah, Categoricity in abstract elementary classes: going up inductive step, Preprint, (100 pages).

[Sh 702] S. Shelah, On what I do not understand (and have something to say), model theory, Math. Japon., 51, (2000), no. 2, 329-377.

[Sh 705] S. Shelah, Toward classification theory of good $\lambda$ frames and abstract elementary classes, In preparation.

[ShVi] S. Shelah and A. Villaveces, Categoricity in abstract elementary classes with no maximal models, Annals of Pure and Applied Logic, 1999.

[Va] M. VanDieren, Categoricity in abstract elementary classes with no maximal models, (57 pages), accepted, subject to revisions by APAL. Preprint available at www.math.1sa.umich.edu/ ${ }^{\mathrm{mvd}} / \mathrm{home} \cdot \mathrm{html}$

[ViZa] Andrés Villaveces and Pedro Zambrano. Hrushovski constructions and tame abstract elementary classes. in preparation.

[Zi] B. Zilber, Analytic and pseudo-analytic structures, Preprint available at www.maths.ox.ac.uk $/{ }^{2}$ zilber

E-mail address, Rami Grossberg: rami@andrew.cmu.edu

Department of Mathematics, Carnegie Mellon University, Pittsburgh PA 15213

E-mail address, Monica VanDieren: mvd@umich.edu

Department of Mathematics, University of Michigan, Ann Arbor Mi 481091109 